\title{
La «pédagogie de la mémoire »de Jean- Paul II sur la base de son premier voyage apostolique en Pologne (1979)
}

Pope Francis, during his first pilgrimage to Poland, in a speech to representatives of the Polish authorities on 27 July 2016 said: «Memory is the hallmark of the Polish people. I was always impressed by Pope John Paul's vivid sense of history. Whenever he spoke about a people, he started from its history, in order to bring out its wealth of humanity and spirituality. A consciousness of one's own identity, free of any pretensions to superiority, is indispensable for establishing a national community on the foundation of its human, social, political, economic and religious heritage»

The author, inspired by the words of Francis, refers to the speeches of John Paul II during his first apostolic journey to Poland (2-10 June 1979), widely recognized as important for the history of Polish, and even Europe. The author discusses the socio-political and religious situation in Poland 1979, and he presents the preparations for the celebration of the 900th anniversary of the death of St. Stanislaus including the role of Karol Wojtyla in them. There is the analysis of selected speeches of John Paul II for his «keen sense of history» and specific pedagogy - «pedagogy of memory». The article ends with a reflection on the impact of the papal visit to Poland on the future of the country and Europe. Historic implications are ubiquitous in the speeches of John Paul II's - vivid in the memory of the Polish Pope and his compatriots - and were to inspire, help Poles in preserving their identity and strengthen in being faithful to God in spite of the temptation of discouragement in the face of painful socio-political conditions. John Paul II recalled that «a man [...] cannot be fully understood without Christ» and that no one has the right to except Christ from His Church and public life. 
Key words: John Paul II, the first apostolic journey to the Polish, pedagogy of memory, Polish religious heritage, the history of Christianity in Poland.

\section{Introduction}

Du 27 au 31 juillet 2016, en l'Année du Jubilé du $1050^{\text {ème }}$ anniversaire du baptême de la Pologne, eut lieu le premier voyage apostolique du Pape François en Pologne, à l'occasion des Journées Mondiales de la Jeunesse. Pendant ces quelques jours passés dans le pays de l'un de ses prédécesseurs Jean-Paul II, François a donné la preuve, dans son enseignement, d'une bonne connaissance de la situation actuelle en Pologne ainsi que du patrimoine du pays.

Dans le discours prononcé le 27 juillet 2016 devant les autorités polonaises, François disait entre autres : «La mémoire caractérise le peuple polonais. Le sens vivant de l'histoire du Pape Jean-Paul II m'a toujours impressionné. Quand il parlait des peuples, il partait de leur histoire pour en faire ressortir les trésors d'humanité et de spiritualité. La conscience de l'identité, libre des complexes de supériorité, est indispensable pour organiser une communauté nationale sur la base de son patrimoine humain, social, politique, économique et religieux, pour inspirer la société et la culture, en les maintenant fidèles à la tradition et en même temps ouvertes au renouveau et à l'avenir $»^{1}$. Le propos de François fait penser à une pédagogie ${ }^{2}$ spécifique de Jean-Paul II - une " pédagogie de la mémoire ». En effet, Jean-Paul II se référait souvent à la mémoire, cette "faculté qui modèle l'identité des êtres humains au niveau tant personnel que collectif $»^{3}$; il évoquait l'histoire des peuples afin d'y puiser des valeurs humaines et spirituelles, inspirer les peuples, les aider à garder leur identité, leur fidélité à la tradition mais aussi une ouverture au renouveau et à l'avenir.

Pour développer la question il faut revenir sur l'enseignement de Jean-Paul II. Étant donné le nombre important de discours prononcés par le Pape polonais, il faut faire un choix. Ce choix tombe sur son

$1 \quad$ François, Rencontre avec les autorités polonaises àl'occasion des JMJ 2016, http:// www.eglise.catholique.fr/actualites/dossiers/jmj-2016/424018-rencontre-avecles-autorites-avec-la-societe-civile-et-avec-le-corps-diplomatique/(03.09.2016).

Nous utilisons le terme «pédagogie » dans le sens d'exercer une influence éducatrice sur l'évolution des autres par un ensemble d'activités et de connaissances éducatives. Por. S. Kunowski, Podstawy wspótczesnej pedagogiki, Łódź 1981, p. 23-24.

$3 \quad$ Cf. Jean-Paul II, Mémoire et identité, Paris 2005, p. 173. 
premier voyage apostolique en Pologne du 2 au 10 juin $1979^{4}$, à l'occasion de l'anniversaire du martyre de saint Stanislaw de Szczepanow Catechetics $(1030-1079)^{5}$. Cette visite est en fait largement considérée comme un événement important, ayant un impact significatif pour la Pologne, voire pour l'Europe ${ }^{6}$.

Le but de cet article n'est pas une analyse exhaustive de tous les aspects possibles de ce pèlerinage ${ }^{7}$ emblématique. Nous essaierons d'en effectuer une relecture sous l'angle des manifestations du « sens vivant de l'histoire » de Jean-Paul II et de sa pédagogie spécifique « pédagogie de la mémoire ${ }^{8}$. Puisqu'il n'y a pas de théologie pratique suspendue dans le vide, détachée de son contexte, nous proposerons, dans un premier temps, de regarder la situation socio-politique et

$4 \quad$ On trouve les interventions officielles de Jean-Paul II en français, prononcées au cours du voyage, entre autres dans la Documentation catholique : Dossier " Le voyage de Jean-Paul II en Pologne », $1^{\mathrm{er}}$ juillet 1979, $\mathrm{n}^{\circ} 1767$ (infra: Dossier) ou dans la publication Le pèlerinage de Jean-Paul II en Pologne, Introduction de V. Volpini, Paris 1979. D. le Corre et M. Sobotka proposent, à leur tour, un dossier du pèlerinage avec quelques extraits des interventions du Pape mais, en revanche, avec plus de commentaires et d'explications concernant le déroulement du voyage, intitulé Jean Paul II en Pologne du 2 au 10 Juin 1979, Bagnolet 1979. Cependant nous n'avons trouvé l'ensemble des interventions du Pape (avec 26 textes absents dans la Documentation catholique) qu'en polonais : Jan Paweł II, Drogowskazy dla Polaków, vol. 2, Kraków 1999, p. 5-200.

Né vers 1030 à Szczepanow, au sud de la Pologne ; assassiné en 1079 de la main du roi Boleslaw II alors qu'il célébrait une messe. Il s'est fait connaître comme défenseur de la justice et de l'ordre moral. Canonisé en 1253 et devenu un des trois Patrons principaux de la Pologne, avec la Vierge Marie Reine de la Pologne et saint Adalbert évêque et martyr (en polonais Wojciech). Pour plus de détails sur la vie et le martyre de saint Stanislaw on peut se référer à un livre jubilaire publié aux États-Unis sous les auspices de l'Association Historique Américano-Polonaise : D. B. Nunis (éd.), Saint Stanislaw, Bishop of Kraków. In commemoration of the 900th Anniversary of His Martyrdom in 1079, Santa Barbara - California 1979.

6 Bernard Lecomte constate qu'il s'agissait, pour Jean-Paul II, « d'ouvrir une brèche dans le mur du totalitarisme, sur des bases historiques et culturelles fortes. Ce n'est pas une démarche politique - le pape n'a jamais lancé et ne lancera jamais de 'croisade' contre le communisme - mais une démarche de vérité. C'est un projet autrement plus fort, plus ambitieux. Plus subversif ». B. Lecomte, Jean-Paul II, Paris 2006, p. 446.

C'est ainsi que l'on appellait souvent les visites du Pape en Pologne. Le Pape lui-même déclarait venir «en pèlerin ».

Au cours de ce premier voyage en Pologne, une centaine de fois Jean-Paul II prononça les mots " mémoire », " souvenir », « commémoration », " se souvenir », et le contenu de ses discours est la meilleure preuve que, pour le Pape, il y a des événements de l'histoire, parmi eux le martyre de saint Stanislaw, que l'on ne peut pas oublier. 
religieuse en Pologne en 1979 afin d'esquisser le contexte dans lequel se déroula ce premier pèlerinage du Pape en sa patrie. Dans un deuxième temps, nous présenterons les circonstances de la préparation et de la célébration de l'anniversaire du martyre de saint Stanislaw et le rôle qu'y joua Karol Wojtyla en tant qu'évêque et pape. Ensuite, nous regarderons « les paroles et les gestes » du Pape sous l'angle de sa «pédagogie de la mémoire ». Pour terminer, nous nous concentrerons sur l'impact de ce voyage sur le lendemain de la Pologne et de l'Europe.

\section{La Pologne et l'Église en Pologne en 1979}

Lannée 1979 en Pologne s'inscrit dans la longue période, complexe et hétérogène, qui a duré de 1945 à 1989. Après la seconde guerre mondiale, la Pologne se trouva sous un nouveau mode d'occupation, à savoir l'emprise de l'Union Soviétique. Ce fut une période d'intenses changements socio-politiques et religieux. Les efforts de soviétisation de la Pologne résultaient du projet de l'URSS visant la création d'une sphère d'influence, d'un bloc de pays communistes. La situation du pays était difficile : les communistes ${ }^{9}$ avaient pour but la consolidation et le renforcement d'un pouvoir populaire, soumis au pouvoir de l'URSS.

Dans les années 1970-1980, c'est Edward Gierek (1913-2001) qui exerce la charge de Premier Secrétaire du Comité Central du Parti Ouvrier Unifié Polonais ${ }^{10}$. Comme son prédécesseur, Wladyslaw Gomulka (1905-1982), qui avait provoqué la déception, voire la haine du peuple, Gierek n'a pas su trouver les moyens d'améliorer la situation dans le pays. Le programme économique a été détaché des réformes politiques, car on ne pensait pas à la démocratisation de la vie politique. Au contraire, les formes telles que la concentration de trop de pouvoirs entre les mains du Premier Secrétaire, la prise de décisions réservée à un groupe étroit de personnes, la confusion de l'appareil du parti avec celui du gouvernement se sont encore développées. Les autorités n'étaient pas compétentes. Après une croissance économique artificielle, car due aux crédits contractés à l'étranger, une crise de plus en plus grave commençait à apparaître à partir de 1975. L'espoir d'une vie meilleure a été déçu, bien que les premières années de l'équipe de Gierek aient semblé prometteuses : augmentation rapide

$9 \quad$ Il s'agit de tous les partisans actifs du nouveau pouvoir, surtout des membres du Parti Ouvrier Polonais, bien que ce parti ne comportât pas que des communistes. Cf. J. Topolski, Historia Polski, Poznań 2007, p. 275.

10 Nom officiel du parti communiste en Pologne depuis 1948 ; en polonais : Polska Zjednoczona Partia Robotnicza (PZPR). 
du niveau de vie, industrialisation du pays, croissance de la natalité. La comparaison entre les conditions de vie en Pologne et en Europe Catechetics occidentale éclairait les Polonais sur leur retard de civilisation. Malgré l'altération de la situation en Pologne, les autorités lançaient la propagande d'un prétendu succès. Après l'augmentation des prix en 1976, de nouveau il y eut des manifestations d'ouvriers à Radom et Ursus, et de nouveau le pouvoir utilisa la répression. Dans ces conditions, une opposition politique avait commencé à naître. Un changement de pensée s'était opéré, dans la mesure où l'on ne réclamait plus la réforme du socialisme, mais l'abandon de ce système. Les nouvelles organisations, les tentatives de création de syndicats indépendants, les publications illégales, l'activité culturelle, les lettres aux autorités annonçaient les changements à venir ${ }^{11}$.

L'Église catholique s'est retrouvée dans ces circonstances difficiles et exigeantes et a dû faire face à l'athéisation. Malgré l'hostilité des autorités communistes envers l'Église et la religion, et peut-être grâce à cela, l'Église est devenue un refuge et une force ; son prestige et son rôle ont augmenté. Parmi les plus grands événements de cette période il faut noter la célébration, en 1966, du millième anniversaire du baptême de la Pologne. Cette célébration, précédée par la Grande Neuvaine du Millénaire (1957-1966), connut un grand succès dans tous les diocèses. Cependant les communistes n'autorisèrent pas la venue en Pologne du Pape Paul VI ${ }^{12}$.

En ce qui concerne la vie religieuse des Polonais, les catholiques, étant la majorité écrasante dans la société, se rendaient en masse aux manifestations religieuses malgré la propagande de l'État. Les initiatives de la base prises par les fidèles désirant la construction d'une église ou d'une chapelle étaient un phénomène caractéristique. $\mathrm{Vu}$ le contexte politique, il est inutile de dire combien cela était difficile. L'enthousiasme, l'astuce et la persévérance des gens vis-à-vis de l'opposition de l'administration publique ont donné des fruits : beaucoup d'églises et de chapelles ont été créées dans des conditions très dures. Il ne faut pas oublier que ces difficultés n'étaient pas dues uniquement à l'administration, mais aussi à la crise économique ${ }^{13}$.

${ }_{11} \quad$ Cf. J. Topolski, Historia Polski, op. cit., p. 287-292.

12 J. Kloczowski, L. Müllerowa, Le christianisme polonais après 1945, [dans :] J. Kloczowski (dir.) Histoire religieuse de la Pologne, Paris 1987, p. 523-524, 537-539. Le Pape Jean-Paul II fera allusion à cette interdiction au cours de son voyage en Pologne. 
L'Épiscopat ne restait pas indifférent aux actions des autorités communistes. Dans les années 1960, 1970 et 1980, il a protesté plusieurs fois contre diverses formes de discrimination : contre la tendance à subordonner la catéchèse aux inspecteurs de l'éducation populaire, la pression sur les personnes qui cédaient un local pour la catéchèse ${ }^{14}$, "les travaux sociaux» (d'intérêt public) organisés les dimanches et les jours de fêtes, les obstacles faits aux pratiques religieuses dans les camps des jeunes, l'action de supprimer les crucifix au nom du caractère laïc de l'école ${ }^{15}$.

Le prestige de l'Église dans la vie nationale et sociale a augmenté. Le « Primat du Millénaire », le cardinal Stefan Wyszynski (1901-1981), s'inscrivit dans la conscience des Polonais comme le défenseur de la dignité de l'homme et en particulier du Polonais, mais aussi de la dignité du travail et des droits de l'homme. Ses initiatives ont montré son identité religieuse et nationale, un christianisme humain et christocentriste, une foi profonde et une piété mariale faisant partie de la tradition chrétienne polonaise ${ }^{16}$.

\section{Jean-Paul II et la célébration de l'anniversaire du martyre de saint Stanislaw}

Pour le printemps 1979, les évêques polonais avaient prévu la célébration du neuf centième anniversaire du martyre de saint Stanislaw. Le jubilé fut soigneusement préparé et l'évêque Karol Wojtyla y eut son apport et ses raisons.

La vie de Wojtyla était liée à Cracovie, d'abord par sa naissance en 1920 (à Wadowice, une cinquantaine de kilomètres de Cracovie), ses études séculières et théologiques, sa première messe dans une crypte de la cathédrale de Wawel, celle qui garde le corps de saint Stanislaw, enfin le ministère d'évêque de Cracovie. En 1964, ce jeune évêque auxiliaire considéré par le pouvoir communiste plutôt comme «un

$14 \quad$ En 1961, la catéchèse a été définitivement exclue de l'école polonaise jusqu'en 1990. Au cours de ces années, elle trouvait sa place dans les «points catéchétiques » : églises, chapelles, locaux paroissiaux, presbytères, maison privées. Cf. B. Skłodowski, Katechizacja w Polsce Ludowej w latach 1945-1990. Zarys problematyki, [dans :] A. Szot (dir.), W małej salce... Katechizacja na Białostocczyźnie w okresie PRL, Białystok 2014, p. 24-27.

15 Cf. E. Majcher, Wokót nauki religii w polskiej szkole, Warszawa 1992, p. 14-16 ; J. Doppke, Katechizacja w Polsce 1945-1990, Pelplin 1998, p. 109-114.

16 Cf. P. Michel, L'Église catholique, [dans :] F. Bafoil (dir.), La Pologne, Paris 2007, p. 379 ; J. Kloczowski, L. Müllerowa, Le christianisme polonais après 1945, art. cit., p. 524-527, 540-541. 
intellectuel, un philosophe, un poète, qu'un 'politique' ${ }^{17}$ et donc facilement accepté sinon proposé par ce pouvoir ${ }^{18}$, remplaça Mgr Eugeniusz Catechetics Baziak après son décès et devint l'archevêque métropolite de Cracovie. Il s'inscrivait ainsi dans l'histoire de l'archidiocèse de Cracovie et celle de ses évêques dont saint Stanislaw est le fameux prédécesseur.

Larchevêque Wojtyla (dès 1967 cardinal Wojtyla) contribua au développement du culte de saint Stanislaw. Il accordait une grande importance à l'anniversaire de son martyre prévu pour le printemps 1979. Il prépara les célébrations pendant sept ans et, en 1978, invita à Cracovie plusieurs cardinaux. Après son élection en tant que pape, le 16 octobre 1978, il souhaitait se rendre en Pologne pour ce jubilé. Ce vœu était explicable : c'est bien lui, qui, en tant que cardinal archevêque de Cracovie, le 8 mai 1972 avait ouvert le synode diocésain. Il souhaitait donc célébrer sa clôture et l'anniversaire du martyre de saint Stanislaw, si soigneusement préparé ${ }^{19}$.

Le Pape savait qu'il ne serait pas facile d'obtenir l'accord des autorités communistes mais il considérait sa participation à cet anniversaire comme un devoir. À la suite des pourparlers le pouvoir communiste autorisa finalement cette première visite du Pape en Pologne et même exprima sa volonté d'accueillir Jean-Paul II dans sa patrie, mais à condition que la visite n'ait pas lieu au mois de mai ${ }^{20}$. Le pouvoir communiste craignait des débordements de sentiments anti-communistes : « La première visite d'un pape en Pologne, pour célébrer le plus violent conflit entre l'Église et l'État dans toute l'histoire polonaise ! Ce n'était pas acceptable ! ${ }^{21}$. Finalement, une visite du 2 au 10 juin fut acceptée et le chef du Conseil d'État, Henryk Jablonski, a invité officiellement le Pape suscitant l'inquiétude du Kremlin. Cette nouvelle date n'a aucunement anéanti les projets du Pape et de l'épiscopat polonais concernant la célébration de l'anniversaire de la mort de saint Stanislaw ${ }^{22}$.

\footnotetext{
17 B. Lecomte, Jean-Paul II, op. cit., p. 261.

18 Cf. Ibidem, p. 259-262.

19 Cf. Ibidem, p. 396, 445.

20 En Pologne, on célèbre la Saint-Stanislaw le 8 mai.

21 B. Lecomte, Jean-Paul II, op. cit., p. 447.

22 Cf. Ibidem, p. 447-448. Pour plus de détails concernant les circonstances de la venue du Pape en Pologne voir:M.Przeciszewski, T. Królak, Demonta $\dot{z}$ « żelaznej kurtyny » rozpocząt się $w$ Gnieźnie, a nie $w$ Berlinie. Rozmowa z metropolita krakowskim kard. Stanistawem Dziwiszem, bytym sekretarzem Jana Pawta II, http://www.opoka.org.pl/biblioteka/T/TH/THO/dziwisz_jp2or201010.html (17.09.2016).
} 
Jean-Paul II s'est bien préparé à son voyage apostolique en sa patrie. Il rédigea seul toutes ses interventions pour enfin, le 2 juin 1979, se rendre en Pologne. Personne ne pouvait prévoir comment se déroulerait ce voyage. Les autorités communistes, mais aussi ecclésiales, craignaient d'éventuels incidents ${ }^{23}$, mais rien de tel n'eut lieu. Quant au Pape, dès son arrivée à Varsovie, il assurait que sa visite " a été dictée par des motifs strictement religieux $»^{24}$. Néanmoins il exprima un souhait qui, tout en restant motivé religieusement, dépassait largement le cadre étroit théologique ou spirituel et révélait une visée politique et sociale ${ }^{25}$ : «Je souhaite aussi vivement que mon présent voyage en Pologne puisse servir la grande cause du rapprochement et de la collaboration entre les nations ; qu'il serve la compréhension réciproque, la réconciliation et la paix dans le monde d'aujourd'hui. Je désire enfin que le fruit de cette visite soit l'unité interne de mes concitoyens, et aussi un nouveau et favorable développement des relations entre l'État et l'Église dans ma chère patrie $»^{26}$.

Les paroles et les gestes du Pape au cours du voyage. Sa «pédagogie de la mémoire »

La première messe de ce voyage apostolique eut lieu le 2 juin 1979, la veille de la Pentecôte, sur la place de la Victoire à Varsovie. Quelque trois cent mille personnes se sont rassemblées ${ }^{27}$. Jean-Paul II, se situant en tant qu' « appelé de la chaire de saint Stanislaw à Cracovie à celle de saint Pierre à Rome ", avouait concevoir son pèlerinage en Pologne pour l'anniversaire du martyre de saint Stanislaw comme un signe $\mathrm{du}$ « pèlerinage polonais à travers l'histoire de l'Église (...) au long des routes ${ }^{28}$ de la Pologne, de l'Europe. Dans ce contexte, le choix d'un pape d'origine polonaise est, pour Jean-Paul II, un signe et un appel pour la Pologne. Il s'agit d'un appel à la responsabilité vis-à-vis de l'avenir, d'un appel au témoignage de foi, à l'exemple de l'appel

$23 \quad$ Le Primat et les évêques polonais avaient préparé les fidèles par des lettres pastorales lues dans les églises. Dans ces lettres, ils considéraient la visite du Pape comme un événement historique, exceptionnel, d'un sens religieux profond. Ils mettaient l'accent sur une préparation spirituelle de tous ainsi que sur une coopération dans tous les préparatifs et pendant le pèlerinage pour que la visite puisse se dérouler en paix et en ordre. Cf. Listy pasterskie Prymasa Polski oraz Episkopatu 1975-1981, Paris 1988, p. 132-134, 138-140, 400-406. Cf. Discours du Pape à l'aéroport de Varsovie, [dans :] Dossier, p. 602. micino en Italie avant de monter dans l'avion. Cf. Dossier, p. 601. Ibidem, p. 602. 
de Jésus adressé à ses apôtres : «vous serez mes témoins » (Ac 1,8). Ce témoignage est celui de la croix et de la résurrection du Christ. Catechetics Le Pape évoquait le jubilé de saint Stanislaw ainsi que le millénaire du baptême de la Pologne pour rappeler « le début de l'histoire de la nation et de l'Église », le jubilé de saint Stanislaw s'inscrivant dans le millénaire du baptême ${ }^{29}$.

En lien avec la liturgie, le Pape évoqua le cénacle et les Apôtres réunis autour de la Vierge Marie et rappela que la Pentecôte est le jour de la naissance de la foi et de l'Église, aussi en terre polonaise. C'est le début du christianisme dans l'histoire, - disait-il - dans la culture, dans les épreuves de la nation polonaise. Ensuite Jean-Paul II prononça les paroles qui seraient citées pendant des années, et qui peuvent bien résumer l'ensemble du pèlerinage : on ne peut pas exclure le Christ de l'histoire de l'homme, on ne peut comprendre sans le Christ ni l'homme ni son histoire ni l'histoire de la Pologne ; le Christ est le critère unique et fondamental. C'est en Jésus Christ que l'histoire de l'homme devient l'histoire du salut, c'est Jésus Christ qui « enseigne la grande cause de l'homme » et qui est comme « un livre ouvert sur l'homme, sur sa dignité, sur ses droits, et en même temps un livre de science sur la dignité et sur les droits de la nation $»^{30}$.

Le Pape a exprimé son souhait que Jésus Christ soit un livre ouvert de la vie pour l'avenir, pour les lendemains polonais. Le lieu de la célébration, à côté de la tombe du Soldat inconnu sur la place de la Victoire, fut l'occasion pour le Pape de mettre dans la bouche du soldat ces paroles : «Il ne peut y avoir d'Europe juste sans l'indépendance de la Pologne $»^{31}$. À la fin de son homélie, Jean-Paul II éleva la voix en priant : «Que descende ton Esprit! Que descende ton Esprit! Et qu'il renouvelle la face de la terre, de cette terre $! »^{32}$.

La première homélie du Pape, prise à titre d'exemple, illustre bien la pédagogie de Jean-Paul II. On pourrait dire qu'il n'a rien dit, mais en même temps il a tout dit, et les Polonais ont tout compris : le Pape n'a rien dit contre le régime, il n'a pas incarné une lutte contre le communisme en tant que tel, il n'a pas appelé les Polonais à une révolution contre les gouvernants. Dans cette homélie, comme dans les autres allocutions et sermons, Jean-Paul II a fait intervenir plusieurs événements de l'histoire gardés dans sa mémoire et celle du peuple polonais. Tout au long de son voyage le Pape a évoqué le baptême

\begin{tabular}{ll}
\hline 29 & Cf. Ibidem \\
30 & Ibidem, p. 608. \\
31 & Ibidem \\
32 & Ibidem, p. 609.
\end{tabular}


de la Pologne (966), le martyre de saint Stanislaw (1079), le martyre de saint Adalbert (997), les vœux du roi Jean II Casimir Vasa (1656) après le « déluge » c'est-à-dire l'invasion des Suédois, les Partages (1795-1918) ou la période de 123 ans où la Pologne n'existait plus sur la carte de l'Europe, la seconde guerre mondiale (1939-1945) avec ses victimes. Il s'est souvent référé aux origines de la Pologne, à ses commencements politiques et religieux, dans le contexte historique polonais indissolubles les uns des autres, depuis le $\mathrm{X}^{\text {ème }}$ siècle $^{33}$, à la tradition, la culture, le patrimoine. Ces « souvenirs historiques » du Pape manifestaient plusieurs aspects, en fonction du lieu de rencontre ou de célébration, des personnes auxquelles le Pape s'adressait ou de la liturgie du jour.

Un des aspects considérables et bien présent dans l'enseignement du Pape était la fidélité de la Pologne à Dieu ${ }^{34}$, exprimée souvent en latin : Polonia semper fidelis. Du coup, si elle l'était semper, elle l'est et elle doit l'être aussi hodie. Cette fidélité à Dieu et le caractère religieux des Polonais, leur pratiques religieuses et leur attachement à la religion et à l'Église tout au long de leur histoire édifient leur identité, l'identité du Polonais-catholique. Cependant cette mémoire de la fidélité de la Pologne à Dieu n'a rien à voir avec un argument de type «c'était toujours comme cela ». Il s'agit de quelque chose de plus profond qui rejoint l'aspect suivant à savoir la souffrance.

La fidélité de la Pologne à Dieu s'est manifestée particulièrement aux moments les plus difficiles - ceux de la souffrance. Encore avant la première messe, à sa rencontre avec les autorités civiles, le Pape a avoué penser « à l'itinéraire historique, grand mais aussi douloureux $»^{35}$ de la Pologne. Ce souvenir de la souffrance, cette memoria passionis $^{36}$, lui permit, entre autres, au milieu des gouvernants communistes, d'exposer des principes de la doctrine sociale de l'Église, les «principes fondamentaux de la justice et de la paix dans le monde contemporain $»^{37}$ pour, précisément, réclamer cette justice et cette

$33 \quad$ Jean-Paul II le rappellera surtout à Gniezno, « le berceau des Piast, origine de l'histoire de la patrie et berceau aussi de l'Église ». Dossier, p. 609-610. Dieu et à l'Église et à sa hiérarchie ». Ibidem, p. 614 . Ibidem, p. 603.

Cette expression est utilisée habituellement pour désigner la Passion du Christ mais il y a des auteurs qui l'appliquent aussi à la souffrance humaine. Cf. J.-B. Metz, La mémoire de la souffrance, facteur de l'avenir. Une forme actuelle de la responsabilité chrétienne, „Concilium” 76 (1972), p. 13. 
paix, valeurs communes indépendamment des convictions politiques ou religieuses.

Cette memoria passionis donna à la visite du Pape aux camps de concentration à Auchwitz et Birkenau, le «Golgotha du monde contemporain », un sens particulier. Jean-Paul II y évoqua Maximilien-Marie Kolbe, Edith Stein, martyrs, ainsi que toutes les victimes des camps de concentration - juifs, russes, polonais. Ce souvenir de la souffrance a poussé le Pape à rappeler l'importance du dialogue, du respect des droits de l'homme et des nations à l'existence et à la liberté. Une nation ne peut asservir l'autre au prix de la conquête, de l'outrage, de l'exploitation, de la mort. À la fin de son homélie prononcée dans cet environnement dramatique, le Pape disait : «Celui [qui prononce ces paroles] est en même temps le fils de la nation qui a subi de la part des autres, au cours de son histoire plus proche de multiples vicissitudes. Il ne le dit pas pour accuser, mais pour rappeler. Il parle au nom de toutes les nations dont les droits sont violés et oubliés. Il le dit parce qu'il y est poussé par la vérité et par la sollicitude pour l'homme ${ }^{38}$.

Ce rappel n'avait pour but d'accuser personne ni de faire de la Pologne une héroïne qui a tellement souffert et qui mérite une compassion, mais d'attirer l'attention du monde entier sur ceux qui souffrent, sur ceux dont les droits ne sont pas respectés. Beaucoup d'observateurs soulignèrent l'importance du fait d'avoir mentionné et les Juifs et les Russes et les Polonais. Encore une fois il s'agissait d'un souvenir qui interroge, qui met en cause l'ordre des choses, qui pousse vers la vérité qui rend libre.

La question du respect des droits fondamentaux que l'histoire peut apprendre, rejoint une autre leçon, celle des valeurs. L'Église est gardienne des valeurs et c'est ainsi qu'elle sert la cause de la paix dans le monde, ainsi et non à travers une activité politique. Le Pape évoqua l'histoire de saint Stanislaw, « patron de l'ordre moral » ${ }^{39}$, pour montrer ensuite l'importance de cet ordre pour l'homme et pour la nation, pour la vie de société.

La mémoire historique de Jean-Paul II, avec ses deux aspects principaux - la fidélité et la souffrance défendant les droits et les valeurs - demande d'être complétée encore par un élément, mentionné mais pas souligné jusqu'à présent, à savoir l'origine polonaise du Pape. La mémoire de cette origine fut souvent manifestée par le Pape lui-même dans ses discours. À plusieurs reprises Jean-Paul II souligna qu'il était « appelé de la chaire de saint Stanislaw à Cracovie à celle de saint

$38 \quad$ Ibidem, p. 634 .

39 Ibidem, p. 621-622. 
Pierre à Rome ». En montrant ce «passage », comme en s'adressant souvent aux fidèles « chers compatriotes » ou « chers fils et filles de la même patrie », Jean-Paul II voulait affirmer que ce lien étroit entre le « cardinal Wojtyla » et sa « chère patrie » n'a pas été dissous au moment de son élection en tant que pape et qu'il n'a pas oublié ses racines, son histoire ${ }^{40}$. L'enjeu de cette affirmation est important : ce n'est pas « un pape » qui rend visite à « un peuple », mais il est bien «l'os de ses os et la chair de sa chair » (cf. Gn 2, 23) et donc il pense comme lui, il ressent comme lui, il connaît ses problèmes, il sait à qui il parle. Jean-Paul II trouva une parole juste pour chacun et pour tous : enfants, jeunesse, adultes, personnes âgées, malades, paysans, ouvriers, scientifiques, artistes, journalistes, gouvernants, prêtres, évêques, séminaristes, religieux. Chacun reçut une parole pertinente, une parole pour lui.

Il faut noter que les participants des rencontres avec le Pape tout au long de son voyage interrompaient souvent ses propos par des applaudissements, chants et acclamations. Jean-Paul II a bien compris ces applaudissements puisqu'il a dit : «Ce qui est important, ce n'est pas qu'ils applaudissent, mais quand ils applaudissent $»^{41}$. Bernard Lecomte a raison de remarquer que c'était « comme un cri libératoire, un signal que tout est devenu possible » et que les lieux de célébrations sont devenus des espaces de liberté ${ }^{42}$. Les Polonais ont pu prendre à cette occasion la mesure de leur cohésion, de leur force : « la base catholique a pris conscience de ce qu'elle représentait le mouvement le plus représentatif dans le pays ${ }^{43}$. Chacun des participants des rencontres avec le Pape en juin 1979 a pu se rendre compte qu'il était entouré par des centaines de milliers de compatriotes formant une unité. Les Polonais en ont pris conscience grâce à cette démonstration. Les Polonais qui disaient « la vérité » dans leurs maisons, en famille ou entre amis, mais jamais en public, se sont rendu compte de leur force et de leur nombre ; ils ont pu expérimenter que le régime n'avait pas tué leur foi, leur unité, leur identité ${ }^{44}$. Ils ont bien compris le message du Pape qui parlait comme "en paraboles », mais dans le contexte

40 À Cracovie, le 6 juin, le Pape disait : «Mon cœur était, et n'a cessé d'être uni à vous, à cette ville, à ce patrimoine ». Ibidem, p. 631.

$41 \quad$ Cité dans : B. Lecomte, Jean-Paul II, op. cit., p. 449.

42 Ibidem

$43 \quad$ P. Michel, L'Église de Pologne et l'avenir de la nation, Paris 1981, p. 142-143.

44 Cf. B. Lecomte, Jean-Paul II, op. cit., p. 449-450. 
donné, chaque «parabole » était compréhensible, son intention et son sens profond lisibles ${ }^{45}$.

Les interventions de Jean-Paul II n'étaient pas des cours d'histoire bien que sa mémoire historique - ou son « sens vivant de l'histoire », comme le disait François - y fût très présente ${ }^{46}$, ce qui peut étonner un lecteur non polonais et ce qui ne sera compréhensible qu'en gardant le souvenir de l'histoire de la Pologne et du christianisme en Pologne ${ }^{47}$. C'étaient finalement des propos profondément religieux, des discours de foi. La pédagogie de Jean-Paul II était une pédagogie de la foi. La clé pour le saisir semblent être les paroles de Jean-Paul II lui-même qui disait : « l'histoire de chaque homme se déroule en Jésus-Christ. En Lui, elle devient l'histoire du salut ${ }^{48}$. C'est pourquoi l'enjeu de cette démarche historique est beaucoup plus important qu'un simple rappel du passé que «nous ne pouvons pas oublier » ${ }^{49}$, pour nous orienter vers l'avenir de la Pologne et de l'Europe, mais il est bien religieux, eschatologique, et aussi anthropologique car « exclure le Christ de l'histoire de l'homme est un acte contre l'homme ${ }^{50}$.

Si le Pape, au cours de son pèlerinage, n'a pas développé un enseignement systématique sur la foi, mais a choisi une démarche historique - « une pédagogie de la mémoire », c'était lié au contexte de l'époque. La foi, dans ces circonstances, était « déjà là ». Les églises en Pologne étaient remplies, les dominicantes nombreux. Cette foi n'était pas à susciter, mais à fortifier et célébrer dans la liturgie. Et

$45 \quad$ Par exemple, le 5 juin, à Czestochowa, dans son allocution aux supérieures religieuses, le Pape a dit par rapport à la nouvelle situation socio-politique dans laquelle se sont trouvées les congrégations religieuses sous le régime communiste (un texte non officiel, disponible en polonais) : «Alors, ce que je dis là, c'est des raccourcis, mais vous savez de quoi il s'agit ». Un autre exemple est l'allocution aux prêtres, du 6 juin. Après avoir évoqué les prêtres qui avaient perdu la vie au cours de la seconde guerre mondiale, le Pape a dit: «Permettez-moi de mettre un frein aux souvenirs qui se pressent dans mon esprit et dans mon cœur ». Dossier, p. 628. Ces souvenirs concernaient sans doute les victimes du régime communiste après la guerre.

46 Quelque 140 fois le Pape prononça le mot «histoire» (en polonais historia, dzieje), quelque 25 fois l'adjectif « historique », de même pour le substantif « héritage »; plus de 200 fois le mot « la Pologne », plus de 300 fois l'adjectif «polonais,-e(s) », une cinquantaine de fois le substantif « le/les Polonais », quelque 230 fois le mot «nation(s)».

47 Le Pape a fait une fois allusion à cette situation « atypique » en Pologne dans son allocution aux évêques polonais. Cf. Dossier, p. 619.

$48 \quad$ Ibidem, p. 608.

$49 \quad$ Ibidem, p. 603.

50 Ibidem, p. 608. 
pourtant les homélies et discours de Jean-Paul II étaient très riches théologiquement et bibliquement. Dans l'ensemble des interventions du Pape apparaissent, avec des accents différents en fonction des circonstances, des éléments de christologie, pneumatologie, ecclésiologie, mariologie, théologie morale, théologie des sacrements, missiologie, doctrine sociale de l'Église ${ }^{51}$.

Il est vrai que l'articulation de la démarche historique avec la démarche théologique n'était pas toujours évidente. Il s'agissait souvent d'une analogie, mais le message était clair et, soulignons-le, tourné vers l'avenir : «Nous irons ensemble sur cette route de notre histoire [...]. Nous irons en nous souvenant du passé, mais l'esprit tendu vers l'avenir... Nous ne retournerons pas au passé ! Nous irons vers l'avenir ! »52.

\section{La réception du message de Jean-Paul II par les Polonais. Le lendemain}

Jean-Paul II n'avait pas peur de puiser dans la mémoire du passé pour inviter ses compatriotes à garder leur identité chrétienne, leur tradition et se tourner vers un meilleur avenir. Ses propos courageux, exprimés avec force, bien que parfois implicite, furent compréhensibles pour les fidèles ainsi que pour les autorités communistes. Ils n'étaient pas neutres par rapport à la situation socio-politique du pays et de l'Europe. Pourtant cette première visite de Jean-Paul II en Pologne n'a pas causé de conflits brutaux entre le pouvoir communiste et la nation. "Tous les observateurs ont été frappés par le recueillement, la discipline, le souci de ne donner prise à aucune manipulation ni provocation de ces rassemblements », soulignait P. Michel ${ }^{53}$. En revanche il y eut beaucoup d'enthousiasme et de vigueur au sein de l'Église durement éprouvée par les événements humains, au sein du peuple polonais invité par le fameux «fils de la même patrie » à une lutte spirituelle, à vaincre le mal par le bien, à porter témoignage ${ }^{54}$.

Pourtant cet enthousiasme fut-il accompagné de gestes concrets ? On reproche parfois aux Polonais une facilité d'avoir applaudi «leur Pape » sans vraiment l'écouter et sans mettre en pratique son

$51 \quad$ Une analyse théologique approfondie des interventions de Jean-Paul II pourrait prolonger et enrichir cette réflexion mais dépasserait largement le cadre de cet article.

$52 \quad$ Dossier, p. 612.

$53 \quad$ P. Michel, L'Église de Pologne et l'avenir de la nation, op. cit., p. 142-143.

54 Cf. V. Volpini, Introduction, [dans :] Le pèlerinage de Jean-Paul II en Pologne, op. cit., p. 9. 
enseignement. Même si parfois c'était vrai, on ne peut pas nier tout ce qui fut un prolongement du premier voyage de Jean-Paul II et une Catechetics réponse des Polonais au message reçu du successeur de saint Pierre et de saint Stanislaw. Les mois et années suivants sont la meilleure preuve que les Polonais ont fait ce que leur avait demandé le Pape, c'est-à-dire ils ont accepté ce « patrimoine spirituel qui a pour nom 'Pologne', avec la foi, l'espérance et la charité que le Christ a placées en nous par le saint baptême » ${ }^{55}$, qu'ils y sont restés fidèles, l'ont conservé, multiplié et transmis ${ }^{56}$. Dans un sondage réalisé en 1980, les Polonais ont exprimé leur attachement au Pape encore autrement : $73 \%$ des répondants ont indiqué Jean-Paul II comme la personne symbolisant le mieux la Pologne ${ }^{57}$.

Une première remarque à faire est le réel intérêt pour les discours du Pape qui s'est manifesté d'une façon très concrète. Or les Polonais, pour suivre le pèlerinage de Jean-Paul II, ne pouvaient pas compter sur les médias de masse officiels, dépendant des autorités communistes qui déformaient l'image du voyage ; d'autres médias ont été censurés. C'est pourquoi beaucoup de fidèles venaient aux rencontres et célébrations présidées par le Pape avec leur magnétophone pour enregistrer ses interventions. Ces enregistrements seront copiés, recopiés et deviendront un matériel soumis aux méditations, discussions dans les paroisses, les associations et les groupes religieux ${ }^{58}$. Les prêtres, encouragés par l'épiscopat, se référeront à l'enseignement du Pape et l'expliqueront dans leurs homélies et sermons. Le message de courage et de confiance sera diffusé, la conscience civique éveillée, l'accent étant mis sur les droits de l'homme et la nécessité de les défendre ${ }^{59}$.

Ensuite il faut évoquer, comme un des fruits de ce pèlerinage, les fameuses «messes pour la Patrie ». L'exemple de l'amour du Pape pour la Pologne et son appel à la fin du pèlerinage « de ne jamais perdre confiance », « de ne pas se laisser abattre » et « de ne pas se décourager $»^{60}$ conduisit le Père Teofil Bogucki (1908-1987), curé de la paroisse Saint-Stanislaw Kostka de Varsovie, à organiser, le dernier dimanche de chaque mois, une « messe pour la Patrie ». L'animation de ces messes, qui rassemblaient de plus en plus de Polonais, fut confiée

\footnotetext{
$55 \quad$ Dossier, p. 643.

$56 \quad$ Cf. ibidem, p. 613.

$57 \quad$ Cf. P. Michel, L'Église catholique, op. cit., p. 378.

$58 \quad$ Cf. B. Lecomte, Jean-Paul II, op. cit., p. 450.

$59 \quad$ Cf. P. Michel, L'Église de Pologne et l'avenir de la nation, op. cit., p. 143.

$60 \quad$ Dossier, p. 643.
} 
au père Jerzy Popieluszko (1947-1984). C'était lui qui choisissait des textes bibliques et poétiques pour chaque messe et disait une homélie. Il n'est pas difficile de deviner que ce choix n'était pas neutre ${ }^{61}$.

Les Polonais ont bien compris le message de Jean-Paul II, ils se sont sentis plus forts grâce à la découverte d'être si nombreux à penser de la même façon, à revendiquer le respect des droits de l'homme. Ils se sont attachés aux problèmes de l'ordre social, économique, politique ; ils revendiquèrent « des espaces de liberté ${ }^{62}$. Les conséquences politiques du voyage de Jean-Paul II comme, plus largement, celles de son élection avec tous les aspects de ce fait (premier pape non italien après de nombreuses années, premier pape slave, venant d'un pays communiste, sa personnalité charismatique et son sens pastoral) font l'objet de plusieurs analyses. Il est difficile d'en trouver une qui lui refuserait un grand rôle que le Pape joua dans les transformations socio-politiques et religieuses, dans la contestation qui prendra de l'ampleur notamment à partir de 1980.

Qu'il suffise, dans ce contexte, d'évoquer une interview intéressante avec le cardinal Stanislaw Dziwisz, menée à l'occasion du trentième anniversaire de ce pèlerinage ${ }^{63}$. L'ancien secrétaire du Pape constate que, effectivement, ce pèlerinage fut le plus important de tous les voyages de Jean-Paul II parce qu'il a inspiré des changements à portée mondiale. La Pologne a changé, les Polonais n'avaient plus peur et ont redressé la tête. Le Pape a libéré la liberté intérieure de l'homme et la vigueur de la nation; dans ce sens on peut dire qu'il a contribué à la naissance de Solidarnosc en 1980. Mais la problématique des discours du Pape dépassait le cadre étroit de sa patrie.

Dans son sermon de Gniezno, le 3 juin 1979, Jean-Paul II disait que sa mission était de faire redécouvrir à l'Europe l'unité entre l'Ouest et $\mathrm{l}^{\prime} E \mathrm{st}^{64}$, et que - comme il ajoutait le 5 juin à Jasna Gora devant les évêques polonais - la responsabilité du christianisme est de « s'engager dans la formation de l'unité spirituelle de l'Europe $»^{65}$. Le cardinal Dziwisz souligne que le Pape a toujours refusé la doctrine du « compromis historique » qui voulait que le marxisme et la « lutte de

${ }_{61} \quad$ Cf. J. Offredo, À Dieu Jerzy !, [dans :] J. Popieluszko, Sermons pour la Patrie. Carnets intimes, Paris 2004, p. VIII ; T. Bogucki, [le titre manque], [dans :] J. Popiełuszko, Kazania patriotyczne, Paris 1984, p. 9. P. Michel, L’Église de Pologne et l'avenir de la nation, op. cit., p. 143. $w$ Gnieźnie, a nie $w$ Berlinie, op. cit. 
classes » fussent considérés comme décisifs pour le développement de l'histoire. Selon le Pape, l'avenir n'appartenait pas au marxisme, Catechetics c'est pourquoi il s'est opposé à introduire le marxisme dans la doctrine sociale de l'Église. Le développement de l'humanité est rendu possible par le respect des droits de la personne, de la liberté, de la possibilité de choisir, de la dignité de l'homme. D'après Dziwisz, le commencement de l'effondrement du mur de Berlin, l'écroulement du rideau de fer, eurent lieu non à Berlin, mais à Gniezno, le 3 juin 1979, dans le discours du Pape. Cet écroulement ne fut que la conséquence du processus débuté en Pologne, en juin 1979. Il serait difficile, après avoir étudié la question, de ne pas donner raison au cardinal.

\section{Conclusion}

En guise de conclusion constatons d'abord une évidence, à savoir l'importance du premier voyage apostolique de Jean-Paul II en Pologne, la portée religieuse, sociale et politique de son enseignement au niveau national et international puisque ce pèlerinage a commencé un processus qui aboutirait à la chute du communisme. Évidemment plusieurs éléments y ont joué un rôle, sans oublier la personnalité extraordinaire de Jean-Paul II et sa " pédagogie de la mémoire ».

Jean-Paul II, dans son enseignement au cours du voyage, n'a à aucun moment appelé ses compatriotes à une révolution contre le régime communiste et n'a accusé personne. Si le Pape a invité les Polonais à une lutte, ce n'était qu'une lutte spirituelle, selon ce que disait saint Paul : " Ne te laisse pas vaincre par le mal, sois vainqueur du mal par le bien $(\mathrm{Rm} \mathrm{12,21})^{66}$. Jean-Paul II, par son pèlerinage, a fait ainsi un geste pastoral et catéchétique. Il est venu rendre témoignage de sa foi, de son espérance et de son amour pour Dieu et l'homme, pour la Pologne et le monde. Il est venu « conforter ses frères dans la foi » (cf. Lc 22, 32), rappeler les principes de l'ordre moral et parler "vrai », montrer la route et guider. Il est venu demeurer avec les siens « au cénacle de la Pentecôte », célébrer la liturgie, prier afin que « l'Église jouisse de la liberté et de la paix dans l'accomplissement de sa mission de salut $»^{67}$.

La «pédagogie de la mémoire » de Jean-Paul II manifestée au cours du voyage apostolique reposait sur le rappel par lui des événements historiques gardés dans la mémoire de la nation pour inspirer les Polonais, les aider à garder leur identité, les fortifier dans la fidélité

\footnotetext{
$66 \quad$ Ibidem, p. 626.

67 Ibidem, p. 617.
} 
à Dieu et dans la persévérance, éviter tout découragement. Grâce à cette pédagogie les Polonais ont cru que la liberté, en tant que l'un des droits fondamentaux, pouvait et devait être assurée, que demain pouvait et devait être meilleur, aussi ce «demain » religieux, politique, social, bref l'avenir de la Pologne, de l'Europe et du monde. Jean-Paul II les a affermis dans la conviction que l'on ne pouvait pas exclure le Christ - «Lumière des nations ${ }^{68}$ et son Église de la vie sociale et politique, l'enjeu étant important : «On ne peut en effet comprendre l'homme à fond sans le Christ $»^{69}$.

En 2005, dans son testament spirituel Mémoire et identité, JeanPaul II dira : " L'histoire de chaque homme et, par son intermédiaire, l'histoire de tous les peuples portent en elles une note eschatologique particulière. [...] Il s'agit d'une lecture de l'histoire, à la lumière de l'Évangile $\gg^{70}$. Ces paroles éclairent la démarche de Jean-Paul II qui demeurait toujours conscient du lien existant entre le monde et l'Église, entre l'histoire de l'humanité et l'histoire du salut ${ }^{71}$. N'estce pas cette conscience qui est la clé de la compréhension du «sens vivant de l'histoire » de Jean-Paul II dont parlait le Pape François devant les autorités polonaises pendant son voyage apostolique en Pologne en juillet 2016 et de ce que nous avons appelé «pédagogie de la mémoire »?

\section{„PEDAGOGIA PAMIĘCI” JANA PAWŁA II NA PRZYKŁADZIE PIERWSZEJ PODRÓŻY APOSTOLSKIEJ DO POLSKI (1979)}

Papież Franciszek, podczas swojej pierwszej pielgrzymki do Polski, w przemówieniu do przedstawicieli polskich władz w dniu 27 lipca 2016 roku, mówił m.in.: "Cechą charakterystyczną narodu polskiego jest pamięć. Zawsze byłem pod wrażeniem żywego zmysłu historycznego Papieża Jana Pawła Il. Gdy mówił o narodach, wychodził od ich dziejów, aby podkreślić ich skarby humanizmu i duchowości. Świadomość tożsamości, wolna od manii wyższości, jest niezbędna dla zorganizowania wspólnoty narodowej na podstawie jej dziedzictwa humanistycznego, społecznego, politycznego, ekonomicznego i religijnego".

Autor artykułu, zainspirowany słowami Franciszka, odwołuje się do przemówień Jana Pawła Il podczas jego pierwszej podróży apostolskiej do Polski

\footnotetext{
68 Cf. Vatican II, Constitution dogmatique sur l'Église «Lumen gentium », n 1 .

$69 \quad$ Dossier, p. 608.

70 Jean-Paul II, Mémoire et identité, op. cit., p. 93-94.

71 Cf. Vatican II, Constitution pastorale sur l'Église dans le monde de ce temps «Gaudium et spes », $\mathrm{n}^{\circ} 1$.
} 
(2-10 czerwca 1979 roku), uznawanej powszechnie za istotną dla dziejów Polski, a nawet Europy. Autor omawia najpierw uwarunkowania społeczno-polityczne Catechetics i religijne w Polsce 1979 roku, po czym przedstawia okoliczności przygotowań do obchodów 900. rocznicy śmierci św. Stanisława z uwzględnieniem roli, jaką odegrał w nich Karol Wojtyła. Następnie zostają przeanalizowane wybrane wypowiedzi Jana Pawła II pod kątem jego „żywego zmysłu historycznego" i specyficznej pedagogii - „pedagogii pamięci”. Artykuł kończy się refleksją nad wpływem papieskiej podróży do Polski na późniejsze losy kraju i Europy. Wszechobecne w przemówieniach Jana Pawła Il wątki historyczne - żywe w pamięci Papieża-Polaka i jego rodaków - miały inspirować, pomagać Polakom w zachowaniu tożsamości oraz umacniać w wierności Bogu wbrew pokusie zniechęcenia wobec bolesnych uwarunkowań społeczno-politycznych. Jan Paweł II przypomniał, że „człowieka [...] nie można do końca zrozumieć bez Chrystusa" i że nikt nie ma prawa wyłączyć Chrystusa i Jego Kościoła z życia publicznego.

Słowa kluczowe: Jan Paweł II, pierwsza podróż apostolska do Polski, pedagogia pamięci, polskie dziedzictwo religijne, historia chrześcijaństwa w Polsce.

\section{Bibliographie:}

1. Bafoil F. (dir.), La Pologne, Paris 2007.

2. Doppke J., Katechizacja w Polsce 1945-1990, Pelplin 1998.

3. Dossier "Le voyage de Jean-Paul II en Pologne », „Documentation catholique", $1^{\text {er }}$ juillet $1979, \mathrm{n}^{\circ} 1767$.

4. François, Rencontre avec les autorités polonaises à l'occasion des JMJ 2016, http://www.eglise.catholique.fr/actualites/dossiers/jmj-2016/424018-rencontre-avec-les-autorites-avec-la-societe-civile-et-avec-le-corps-diplomatique/ (03.09.2016).

5. Jan Paweł II, Drogowskazy dla Polaków, vol. 2, Kraków 1999.

6. Jean-Paul II, Mémoire et identité, Paris 2005.

7. Kloczowski J., Müllerowa L., Le christianisme polonais après 1945, [dans :] J. Kloczowski (dir.), Histoire religieuse de la Pologne, Paris 1987, p. 497-553.

8. Kunowski S., Podstawy wspótczesnej pedagogiki, Łódź 1981.

9. Le Corre D., Sobotka M., Jean Paul II en Pologne du 2 au 10 Juin 1979, Bagnolet 1979.

10. Le pèlerinage de Jean-Paul II en Pologne, Introduction de Valerio Volpini, Paris 1979.

11. Lecomte B., Jean-Paul II, Paris 2006.

12. Listy pasterskie Prymasa Polski oraz Episkopatu 1975-1981, Paris 1988.

13. Majcher E., Wokót nauki religii w polskiej szkole, Warszawa 1992.

14. Metz J.-B., La mémoire de la souffrance, facteur de l'avenir. Une forme actuelle de la responsabilité chrétienne, „Concilium” 76 (1972), p. 9-25.

15. Michel P., L'Église catholique, [dans :] F. Bafoil (dir.), La Pologne, Paris 2007, p. 371-394. 
16. Michel P., L'Église de Pologne et l'avenir de la nation, Paris 1981.

17. Nunis D. B. (éd.), Saint Stanislaw, Bishop of Kraków. In commemoration of the 900th Anniversary of His Martyrdom in 1079, Santa Barbara - California 1979.

18. Offredo J., À Dieu Jerzy !, [dans :] J. Popieluszko, Sermons pour la Patrie. Carnets intimes, Paris 2004.

19. Popiełuszko J., Kazania patriotyczne, Paris 1984.

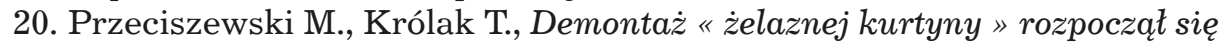
$w$ Gnieźnie, a nie $w$ Berlinie. Rozmowa z metropolita krakowskim kard. Stanistawem Dziwiszem, bytym sekretarzem Jana Pawta II, http:/www. opoka.org.pl/biblioteka/T/TH/THO/dziwisz_jp2or201010.html (17.09.2016).

21. Skłodowski B., Katechizacja w Polsce Ludowej w latach 1945-1990. Zarys problematyki, [dans :] A. Szot (dir.), W matej salce... Katechizacja na Białostocczyźnie w okresie PRL, Białystok 2014, p. 17-30.

22. Topolski J., Historia Polski, Poznań 2007.

23. Vatican II, Constitution dogmatique sur l'Église "Lumen gentium » (21.11.1964).

24. Vatican II, Constitution pastorale sur l'Église dans le monde de ce temps «Gaudium et spes »(08.12.1965). 